\title{
Diagnosis reliability of combined flexible sigmoidoscopy and fecal-immunochemical test in colorectal neoplasia screening
}

This article was published in the following Dove Press journal:

OncoTargets and Therapy

4 November 2016

Number of times this article has been viewed

\section{Dana lovanescu' \\ Mirela Frandes ${ }^{2}$ \\ Diana Lungeanu ${ }^{2}$ \\ Amelia Burlea ${ }^{3}$ \\ Bogdan P Miutescu ${ }^{4}$ \\ Eftimie Miutescu' \\ 'Department of Gastroenterology, Faculty of Medicine, Pharmacy and Dental Medicine, "Vasile Goldis" West University of Arad, "Department of Functional Sciences, "Victor Babes" University of Medicine and Pharmacy of Timisoara, ${ }^{3}$ Department of Pathology, County Hospital of Arad, ${ }^{4}$ Department of Gastroenterology, "Victor Babes" University of Medicine and Pharmacy of Timisoara, Romania}

Correspondence: Diana Lungeanu Department of Functional Sciences, "Victor Babes" University of Medicine and Pharmacy Timisoara, Piata Eftimie Murgu 2, 30004I Timisoara, Romania Tel +40 722488775

Fax +40256490288

Email dlungeanu@umft.ro
Background: Employing colonoscopy, the gold standard in colorectal cancer (CRC) diagnosis testing, for CRC screening presents a significant risk of complications. Alternative methods with a lower invasive-level and fewer risks are proposed in combination, though each with lower diagnosis performance when applied separately. The main objective of this cross-sectional pilot study was to evaluate the feasibility of a CRC screening program using combined flexible sigmoidoscopy and fecal-immunochemical test (FIT).

Methods: The patient population consisted of 2,201 consecutive-case symptomatic patients attending the gastroenterology outpatient clinic with mild complaints between 2012 and 2014 . They were referred for FIT. A sample of 252 individuals underwent a subsequent colonoscopy, blind to FIT results, and theoretical sigmoidoscopy was simulated. On a subsample of 57 patients, real sigmoidoscopy was additionally performed. Prior probabilities in terms of patients' compliance and CRC prevalence were estimated, together with predictive ability of FIT and sigmoidoscopy in screening population. We assessed the merit of a screening strategy employing two-stage serial multiple testing: a) first stage by combining two parallel tests, that is, flexible sigmoidoscopy and FIT and b) colonoscopy as the second diagnosis test. The scheme was validated using the actual predictive values derived from the study population.

Results: Colonoscopy found $75(29.76 \%)$ individuals with advanced neoplasia. FIT was positive in $30.3 \%$ of advanced neoplasia cases, while between $23.73 \%$ and $28.28 \%$ met the theoretical sigmoidoscopy simulation criteria, with good concordance between real and theoretical sigmoidoscopy. The colonoscopy referral compliance rate was 52\% among FIT-positives. Sensitivity and specificity of the first-stage test combination were better than sigmoidoscopy alone (McNemar test: $P<0.001)$. Negative predictive values for low prevalence levels were between $81.5 \%$ and $90.12 \%$.

Conclusion: Combining less resource challenging and less invasive testing procedures is worthwhile in colorectal neoplasia detection, improving sensitivity and specificity of either test alone, and leading to better posterior probabilities in usual screening scenarios.

Keywords: colorectal cancer, test combination, predicted values, screening

\section{Introduction}

Colorectal cancer (CRC) is a major health issue worldwide, ${ }^{1}$ so any form of CRC screening is effective and cost saving even in an average risk population. ${ }^{2}$ The US Preventive Services Task Force recommended screening for CRC using high-sensitivity fecal occult blood testing, flexible sigmoidoscopy (SIG) with interval fecal occult blood testing, or colonoscopy (COL). ${ }^{3,4} \mathrm{COL}$ is the gold standard for colon examination, although it also is a complex and invasive procedure with a small, but not 
insignificant risk of major complications. When deciding which test to use, several factors should be considered, for example, availability of endoscopy units and the burden on their capacity, available human resources (specialist medical doctors, nurses, and laboratory staff). Though flexible SIG can only examine the distal part of the colon, the procedure is less time consuming, no sedation is needed, and the bowel preparation is simpler. On the other hand, when a polyp or an adenoma (depending on the chosen medical criteria) is detected during the examination, a subsequent COL might nevertheless be required. Fecal-immunochemical tests (FITs) have gradually replaced chemical-based tests, showing an improved sensitivity for advanced neoplasia.

Mixed criteria were proposed to classify the SIG results, namely: a) USPLCO criteria proposed by the USA Prostate, Lung, Colorectal and Ovarian (PLCO); ${ }^{5}$ b) UK flexible SIG criteria; ${ }^{6}$ c) Italian SCORE trial criteria; ${ }^{7}$ and d) Norwegian NORCCAP trial criteria. ${ }^{8}$

There has been an upward trend in CRC mortality in Europe, for example, by a median of $60 \%$ for men between 1989 and 2011. Though it decreased by a median of $14.7 \%$ for women, over the same period, the trend in CRC mortality is still upward in the Central-Eastern European countries. ${ }^{9}$ So combining less invasive tests has been suggested. ${ }^{10}$

This paper originates from a feasibility study aiming to evaluate the best screening strategy for colorectal neoplasia in western Romania. The primary objective was to assess a screening strategy employing multiple testing in a two-stage serial scenario: a) a first stage combining two parallel tests, that is, FIT and flexible SIG, both insufficiently effective when used alone and b) a second stage using COL as the subsequent diagnosis test, in cases of positive first-stage findings. In addition, we explored the patients' compliance and the CRC prevalence in the study population, providing an estimate for the prior probabilities. Subsequently, we evaluated the predictive ability of FIT and SIG in the study population, and compared the performance with the values found in the literature. Finally, the proposed scheme was validated by comparing the theoretical values with the actual predictive values derived from the study population.

\section{Methods}

The study protocol was approved by Ethics Committee for Scientific Research of the "Vasile Goldis" Western University of Arad. The County Hospital of Arad is a teaching hospital for the Faculty of Medicine, Pharmacy and Dental Medicine. The costs of medical investigations were covered by the medical insurance packages and the hospital (in part), or by the patients themselves. Each patient signed an informed consent to participate.

\section{Patient population}

The patient population consisted of consecutive-case symptomatic patients attending the Gastroenterology outpatient clinic in the County Hospital of Arad for mild abdominal complaints, between January 2012 and December 2014. The inclusion criteria in this cross-sectional study were the following: absence of any suggestive CRC symptoms, and being aged between 40 and 79 years. Subjects were excluded if they reported recent explorations (eg, SIG or COL in the last 5 years) or one of the following was present: they had a personal history of CRC; they had a family history of hereditary or familial CRC (defined as $\geq 2$ first-degree relatives with CRC or one relative CRC-diagnosed before the age of 60 years); they had a terminal medical condition.

All the 2,201 patients who complied with the study criteria were referred for FIT (Figure 1 shows the study flowchart). A sample of 252 individuals, that is, arm (A), underwent a subsequent COL (blind to the FIT results), and a theoretical SIG was simulated. On a subsample of 57 patients, that is, arm (B), a real SIG was additionally performed in order to explore the simulated SIG bias. The sensitivities and specificities were determined both for the single tests and their combinations, followed by a reliability analysis.

\section{FIT investigation}

All individuals collected one stool sample without specific diet or medication restrictions. The rapid immunochemical test Hem-Check 1 (VedaLab, France) was used. Hem-Check is an immunochromatographic analysis with a sensitivity range between 0.04 and $120 \mu \mathrm{g} \mathrm{Hb} / \mathrm{g}$ of feces. The standard FIT cutoff ( $\geq 20 \mu \mathrm{g} \mathrm{Hb} / \mathrm{g}$ ) was considered for the fecal hemoglobin concentration.

\section{Colonoscopy}

Before COL, all patients were given written instructions regarding the required diet (a low-residue diet during the entire day before the investigation), and the colonic cleansing protocol. A split-dose bowel cleansing regimen of $4 \mathrm{~L}$ polyethylene glycol-electrolyte lavage solution was used, and propofol deep sedation. The patients were examined with a videocolonoscope Olympus CF-HQ190 Evis Exera III. All polyps detected during the COL were endoscopically removed and retrieved for subsequent histological examination. If a colon cancer was detected, biopsies were taken. The examination exclusion criteria consisted of incomplete COL, poor bowel preparation, or lack of histology. 


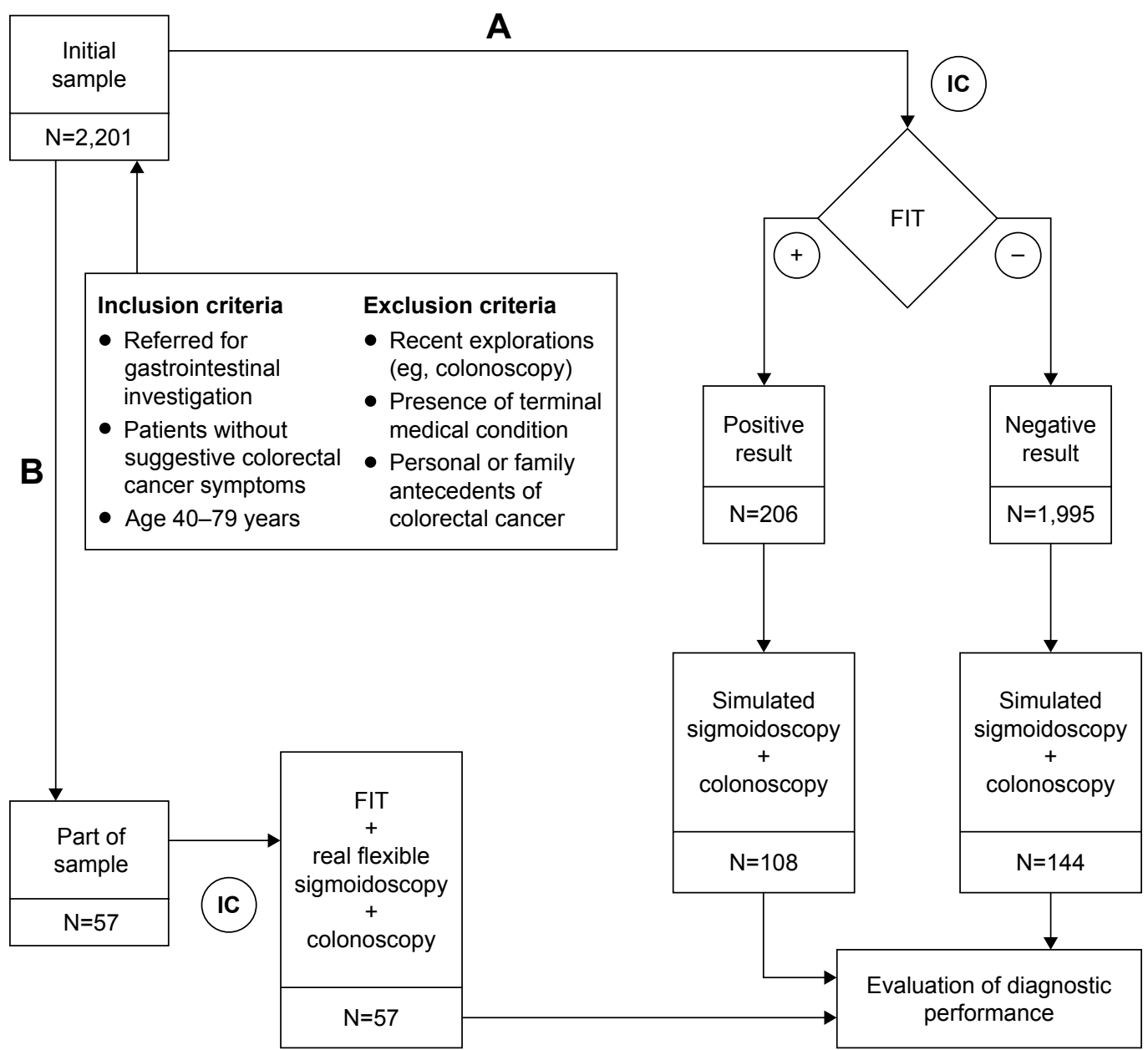

Figure I Study flow diagram. Arm (A) includes the individuals referred for FIT and subsequent colonoscopy; the arm (B) subsample consists of the individuals who additionally underwent real sigmoidoscopy.

Abbreviations: FIT, fecal-immunochemical test; IC, informed consent.

\section{Simulated SIG}

SIG was simulated considering COL findings in the rectum, the sigmoid, and the descending colon (distal to the splenic flexure), in a similar manner to Castro et al. ${ }^{11}$ The diagnostic yield and the theoretical post-SIG referral for COL were defined according to four already established sets of criteria: a) USA PLCO trial, where any polyp (either adenoma or not) would be referred; ${ }^{5}$ b) UK flexible SIG, where any distal polyp $\geq 10 \mathrm{~mm}$, tubulovillous or villous histology, high-grade dysplasia CRC, or $\geq 20$ polyps above the distal rectum would be referred; ${ }^{6}$ c) SCORE trial, where any distal polyp $\geq 5 \mathrm{~mm}$, tubulovillous or villous histology, high-grade dysplasia, $\geq 3$ adenomas, or CRC would be referred $;{ }^{7}$ and d) NORCCAP trial, where any distal polyp $\geq 10 \mathrm{~mm}$, any adenoma, or CRC would be referred. ${ }^{8}$

In addition, we simulated the diagnostic performance of FIT (actual results) in combination with each simulated flexible SIG strategy.

\section{Real flexible SIG}

Before the SIG, a bowel preparation was performed, consisting of a single enema self-administered either at home or at the endoscopy unit.

\section{Advanced neoplasia}

Advanced neoplasia was defined as cancer or adenomas $>10 \mathrm{~mm}$, presenting villous architecture, or manifesting high-grade dysplasia.

\section{Data analysis}

Descriptive statistics were presented as mean \pm standard deviation for continuous variables, or frequency counts with the percentage for the categorical variables. Exploratory statistical testing was applied to check whether the positive and negative subjects' groups were comparable regarding their age, sex, or urban or rural living (Mann-Whitney $U$-test for continuous variables and chi-square or Fisher exact test 
for categorical ones). Sensitivity (SN) and specificity (SP) were calculated for individual diagnosis tests, that is, FIT and flexible SIG, based on the COL result as the gold-standard reference. For comparing the binary diagnostic tests' performance, the exact McNemar statistical test for correlated proportions was applied.

To assess the performance of test combination, two scenarios were considered, as follows.

\section{Parallel design}

The tests are administered independently and at the same time, and the results are logically combined, result $\mathrm{C}$ being positive if at least one test is positive, as in Equation (1); the effect is a higher sensitivity (ie, decreased false negative rate) at the cost of a lower specificity.

$$
\begin{gathered}
C_{+}=T_{1+} \cup T_{2+} \cup \ldots \cup T_{S_{+}} \\
S N\left(T_{C}\right)=\sum_{i=1}^{S} S N\left(T_{i}\right)+\prod_{i=1}^{S} S N\left(T_{i}\right)-\left(\sum_{i=1}^{S} S N\left(T_{i}\right)\right)^{2} \\
+\sum_{i=1}^{S}\left(S N\left(T_{i}\right)\right)^{2} \\
S P\left(T_{C}\right)=\prod_{i=1}^{S} S P\left(T_{i}\right)
\end{gathered}
$$

when the number of combined tests is $S=2$, equations for the parallel design become:

$$
\begin{aligned}
S N\left(T_{C}\right)= & S N\left(T_{1}\right)+S N\left(T_{2}\right)-S N\left(T_{1}\right) \times S N\left(T_{2}\right) \\
= & S N\left(T_{1}\right)+S N\left(T_{2}\right) \times\left[1-S N\left(T_{1}\right)\right] \\
& S P\left(T_{C}\right)=S P\left(T_{1}\right) \times S P\left(T_{2}\right)
\end{aligned}
$$

\section{Serial design}

Each test is administered taking into consideration the previous testing results; if the first result is negative, the final result is declared negative, and no other test is applied; otherwise, the procedure is repeated up to the number of available tests, as in Equation (4); this improves specificity (ie, decreased false positive rate) at the cost of a lower sensitivity.

$$
\begin{gathered}
C_{-}=T_{1-} \cup T_{2-} \cup \ldots \cup T_{S-} \\
S N\left(T_{C}\right)=\prod_{i=1}^{S} \operatorname{SN}\left(T_{i}\right)
\end{gathered}
$$

$$
\begin{aligned}
S P\left(T_{C}\right)= & \sum_{i=1}^{S} S P\left(T_{i}\right)+\prod_{i=1}^{S} S P\left(T_{i}\right)-\left(\sum_{i=1}^{S} S P\left(T_{i}\right)\right)^{2} \\
& +\sum_{i=1}^{S}\left(S P\left(T_{i}\right)\right)^{2}
\end{aligned}
$$

when the number of combined tests is $S=2$, equations for the serial design become:

$$
\begin{gathered}
S N\left(T_{C}\right)=S N\left(T_{1}\right) \times S N\left(T_{2}\right) \\
S P\left(T_{C}\right)=S P\left(T_{1}\right)+S P\left(T_{2}\right)-S P\left(T_{1}\right) \times S P\left(T_{2}\right) \\
=S P\left(T_{1}\right) \times\left[1-S P\left(T_{2}\right)\right]+\operatorname{SP}\left(T_{2}\right)
\end{gathered}
$$

Figure 2 shows the two-stage screening scheme proposed and analyzed.

The statistical analysis was performed using SPSS version 17.0 and $\mathrm{R}$ project packages for statistical computing. Confidence level of 0.95 was considered for the estimating intervals, and a $P$-value of 0.05 was the threshold for the statistical significance.

\section{Results}

The initial sample $(\mathrm{N}=2,201)$ included individuals aged from 40 to 79 years $(61.33 \pm 9.805), 39.3 \%$ females, and $75.1 \%$ from urban area. We found no statistically significant differences in proportion between subjects with positive and negative FIT results regarding either the sex (chi-square test, $P=0.18$ ), or urban/rural living (chi-square test, $P=0.961$ ).

The sample in arm (A) sample included $\mathrm{N}=252$ individuals aged 40-79 years (63.65 \pm 8.781$)$. The main characteristics are presented in Table 1. We found no statistically significant differences in proportion between subjects with and without lesions regarding the sex (chi-square test, $P=0.716$ ), age (Mann-Whitney $U$-test, $P=0.51$ ), or urban/rural living (chi-square test, $P=0.083$ ).

Proportion of advanced neoplasia was $3.4 \%$ of the total of 2,201 patients, with $2 \%$ CRC. The compliance with the COL referral after a positive FIT was rather low, that is, 108 out of 206 total of FIT-positives $(52 \%)$. Out of the 252 subjects who actually underwent a colonoscopic investigation, $75(29.76 \%)$ individuals tested positive for advanced neoplasia (Table 1).

\section{Advanced neoplasia}

Diagnostic performance of the testing approaches is presented in Table 2. FIT alone proved poor sensitivity for 


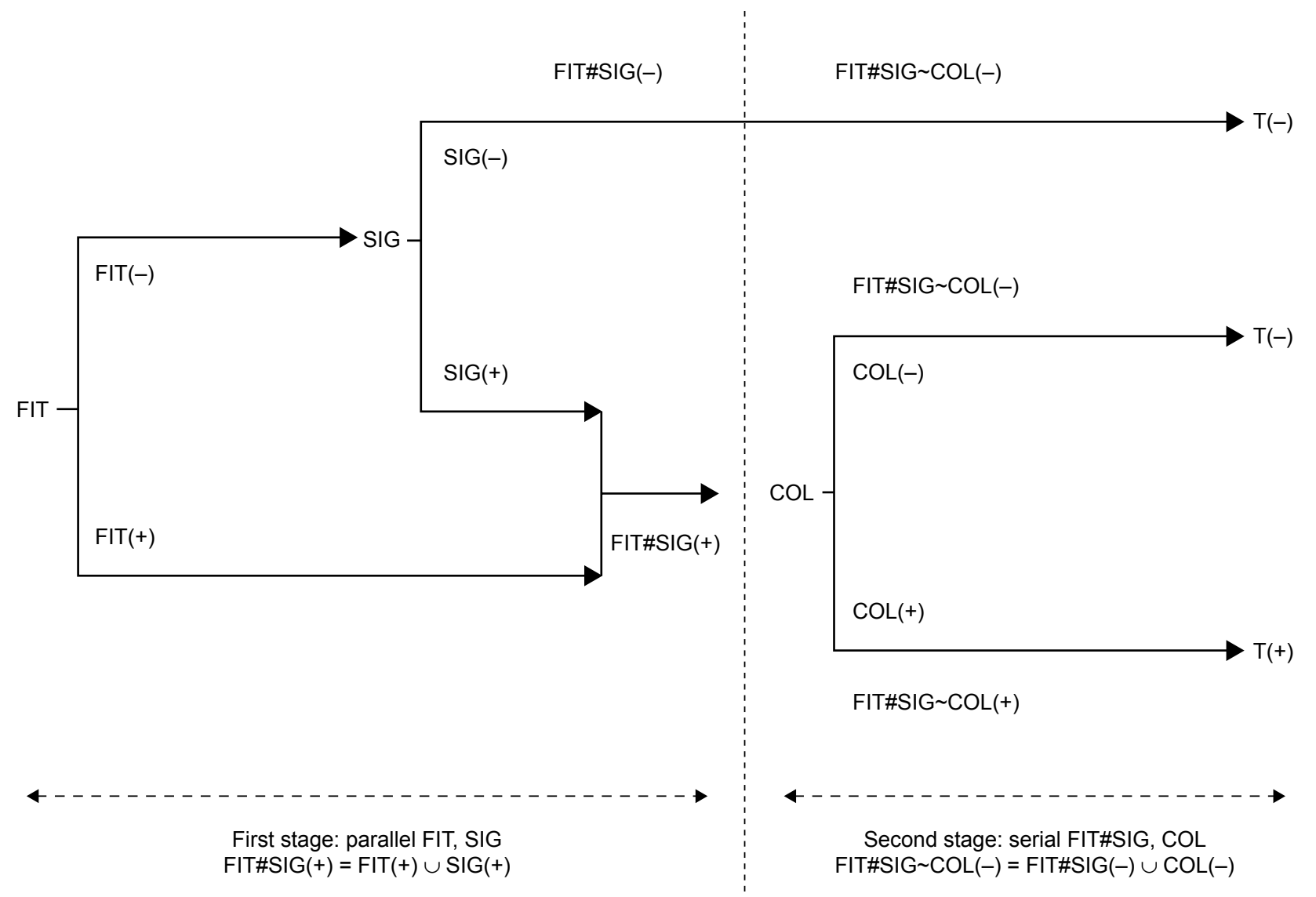

Figure 2 Scheme for testing combination.

Notes: The first stage consists of the parallel combination (notation \#) of two diagnostic tests. The second stage is a serial combination (notation ) of the previous stage result and a third reference (gold standard) test. $\mathrm{T}( \pm)$ denotes the final decision in the screening process.

Abbreviations: COL, colonoscopy; FIT, fecal-immunochemical test; SIG, sigmoidoscopy.

Table I Characteristics of individuals included in the evaluation of diagnosis reliability

\begin{tabular}{ll}
\hline Characteristics of the patients & Number (N=252) \\
\hline Age (years) & $63.65 \pm\left. 8.78\right|^{\mathrm{a}}$ \\
Sex (female) & $144(57.1 \%)$ \\
Urban (yes) & $175(69.4 \%)$ \\
Colorectal cancer (yes) & $45(17.85 \%)$ \\
Distal to splenic flexure & $24(9.52 \%)$ \\
Proximal to splenic flexure & $16(6.35 \%)$ \\
Distal and proximal to splenic flexure & $5(1.98 \%)$ \\
Advanced neoplasia (yes) & $75(29.76 \%)$ \\
Distal to splenic flexure & $39(15.48 \%)$ \\
Proximal to splenic flexure & $26(10.32 \%)$ \\
Distal and proximal to splenic flexure & $10(3.97 \%)$ \\
Advanced adenoma (yes) & $30(10.37 \%)$ \\
Distal to splenic flexure & $15(3.97 \%)$ \\
Proximal to splenic flexure & $10(2.52 \%)$ \\
Distal and proximal to splenic flexure & $5(1.98 \%)$ \\
Nonadvanced adenoma (yes) & $57(22.62 \%)$ \\
Proximal to splenic flexure & $23(9.13 \%)$ \\
Distal and proximal to splenic flexure & $10(3.97 \%)$ \\
\hline
\end{tabular}

Note: aMean \pm standard deviation. nonadvanced adenoma (Table 2A). The simulated SIG was rather poor as well, especially in terms of sensitivity, that is, leading to a large proportion of false negatives (Table 2B).

Table $2 \mathrm{C}$ synthesizes the validation results in arm (B), as in Figure 1. Although point estimates for sensitivity and specificity varied, the $95 \%$ confidence intervals overlapped to a large degree and the exact McNemar statistical test for correlated proportions resulted in $P$-value asymptotically reaching 1 , so we considered arm (B) to be a valid proof for the screening evaluation conducted by using theoretical SIG simulation. As the approach, employing UK criteria ${ }^{6}$ was the most conservative in terms of diagnosis performance; all further analyses for test combination employed their sensitivity and specificity values.

\section{Proximal advanced neoplasia}

For advanced proximal neoplasia in the sample of arm (A), the FIT sensitivity was $75 \%$, while the specificity was $69.17 \%$ (Table 2A). 
Table 2 Diagnostic performance of the different testing approaches

\begin{tabular}{|c|c|c|c|c|c|c|}
\hline Diagnosis method & Positives & Negatives & Sensitivity (\%) ${ }^{a}$ & Sensitivity $(95 \% \mathrm{Cl})$ & Specificity (\%) ${ }^{a}$ & Specificity $(95 \% \mathrm{Cl})$ \\
\hline Colonoscopy & 75 & 177 & Reference test & & & \\
\hline \multicolumn{7}{|c|}{ A. FIT for advanced and non-advanced neoplasia detection $(\mathrm{N}=252)$} \\
\hline AN & 91 & 104 & 72 & $(60.44,81.76)$ & 69.17 & $(60.09,77.27)$ \\
\hline Proximal AN & 64 & 92 & 75 & $(57.8,92.21)$ & 69.17 & $(60.09,77.27)$ \\
\hline Distal AN & 70 & 99 & 67.35 & $(52.46,80.05)$ & 69.17 & $(60.09,77.27)$ \\
\hline Non-AA & 50 & 127 & 23.81 & $(8.22,47.17)$ & 71.15 & $(63.37,78.12)$ \\
\hline \multicolumn{7}{|c|}{ B. Simulating sigmoidoscopy strategies for advanced neoplasia detection $(\mathrm{N}=252)$} \\
\hline USPLCO ${ }^{\mathrm{b}}$ & 58 & 137 & 68 & $(56.22,78.31)$ & 94.17 & $(88.35,97.62)$ \\
\hline $\mathrm{UK}^{\mathrm{b}}$ & 56 & 139 & 57.33 & $(45.38,68.69)$ & 89.17 & $(82.19,94.1)$ \\
\hline SCORE $^{\mathrm{b}}$ & 60 & 135 & 62.67 & $(50.73,73.57)$ & 89.17 & $(82.19,94.1)$ \\
\hline NORCCAPb & 66 & 137 & 66.67 & $(54.83,77.14)$ & 93.33 & $(87.29,97.08)$ \\
\hline \multicolumn{7}{|c|}{ C. Comparison of real and simulated sigmoidoscopy, on a sub-sample $(\mathrm{N}=57)$} \\
\hline Real SIG & 7 & 35 & 40.74 & $(19.92,65.11)$ & 93.33 & $(73.47,97.89)$ \\
\hline USPLCO ${ }^{\mathrm{b}}$ & 7 & 35 & 41.67 & $(15.17,72.33)^{c}$ & 93.33 & $(77.93,99.18)^{c}$ \\
\hline $\mathrm{UK}^{\mathrm{b}}$ & 6 & 36 & 25 & $(5.49,57.19)^{c}$ & 90 & $(73.47,97.89)^{c}$ \\
\hline SCORE & 7 & 35 & 25 & $(5.49,57.19)^{c}$ & 86.67 & $(69.28,96.24)^{c}$ \\
\hline NORCCAPb & 9 & 33 & 41.67 & $(15.17,72.33)^{c}$ & 86.67 & $(69.28,96.24)^{c}$ \\
\hline
\end{tabular}

Notes: Colonoscopy was the reference test for FIT and for the simulating sigmoidoscopy strategies. Methods for simulation: USA PLCO trial; ${ }^{5}$ UK flexible sigmoidoscopy trial;; SCORE trial; $;$ NORCCAP trial. ${ }^{8}$ aValues are expressed as point estimates and $95 \%$ Cls. ${ }^{b}$ Rectum, sigmoid, and descending colon were considered for sigmoidoscopy simulation. 'Exact McNemar test did not reject the null hypothesis when compared with Real SIGmoidoscopy.

Abbreviations: AA, advanced adenoma; AN, advanced neoplasia; $\mathrm{Cl}$, confidence interval; FIT, fecal-immunochemical test.

\section{Real flexible SIG}

The arm (B) subsample ( $\mathrm{N}=57)$ included individuals aged from 44 to 77 years $(63.28 \pm 8.647), 57.9 \%$ of whom were females, and $71.9 \%$ of whom were living in an urban area. The sensitivity and specificity of flexible SIG for advanced neoplasia detection was $40.74 \%$ and $93.33 \%$, respectively (Table 2C). Although in real flexible SIG, the splenic flexure could not always be reached, or the colon preparation had not always been done correctly, the sensitivity was nevertheless reasonably good and comparable to the simulated approach by USPLCO and NORCCAP criteria.

\section{Test combination}

The pilot scheme proposed for CRC screening is presented in Figure 2. It consists of a two-stage serial design with combination of two parallel tests, that is, SIG and FIT, in the first stage and a single gold-standard test, that is, COL, in the second stage. The parallel combination of the first-stage increases the sensitivity, resulting in a lower false negative rate. The first stage takes advantage of the complementary capacity of SIG and FIT to detect advanced distal and proximal neoplasia, respectively. In the second stage, the specificity increases while maintaining a reasonably high sensitivity. Overall, for such a serial testing scheme, when both FIT and SIG are negative in the first stage, that is, FIT\#SIG(-), the final screening result FIT\#SIG COL(-) is negative, thus reducing the number of unnecessary COL tests.

The performance issue lies in the false negative rate, that is, whether or not we can actually afford to apply such a screening scheme. Table 3 synthesizes the performance of the first-stage FIT\#SIG combination, as in Figure 2, with simulated SIG.

The interpretation of any diagnostic test depends not only on sensitivity and specificity, but also on the baseline prior

Table 3 Parallel combination of FIT and sigmoidoscopy, as the first stage of the screening scheme proposed (N=252)

\begin{tabular}{|c|c|c|c|c|c|c|c|}
\hline Combination & Positives & Negatives & Sensitivity $(\%)$ & Sensitivity $(95 \% \mathrm{Cl})$ & $P$-value ${ }^{b}$ & Specificity $^{\mathrm{a}}(\%)$ & Specificity $(95 \% \mathrm{Cl})$ \\
\hline FIT\#USPLCO' & 110 & 85 & 96 & $(88.75,99.17)$ & $<0.001$ & 68.33 & $(59.22,76.52)$ \\
\hline FIT\#UKc & 105 & 90 & 89.33 & $(80.06,95.28)$ & $<0.001$ & 68.33 & $(59.22,76.52)$ \\
\hline FIT\#SCORE & 106 & 89 & 90.67 & $(81.71,96.16)$ & $<0.001$ & 68.33 & $(59.22,76.52)$ \\
\hline FIT\#NORCCAPc & 107 & 88 & 93.33 & $(85.12,97.8)$ & $<0.001$ & 69.17 & $(60.09,77.27)$ \\
\hline
\end{tabular}

Notes: Simulated sigmoidoscopy was performed based on colonoscopy. Methods for simulation: USA PLCO trial; ${ }^{5}$ UK flexible sigmoidoscopy trial; 6 SCORE trial; 7 NORCCAP trial. ${ }^{8}$ Values are expressed as point estimates and $95 \%$ Cls. ${ }^{b}$ Significance of combination sensitivity when compared with sigmoidoscopy alone (McNemar test). ${ }^{~}$ Rectum, sigmoid, and descending colon were considered for sigmoidoscopy simulation.

Abbreviations: $\mathrm{Cl}$, confidence interval; FIT, fecal-immunochemical test. 

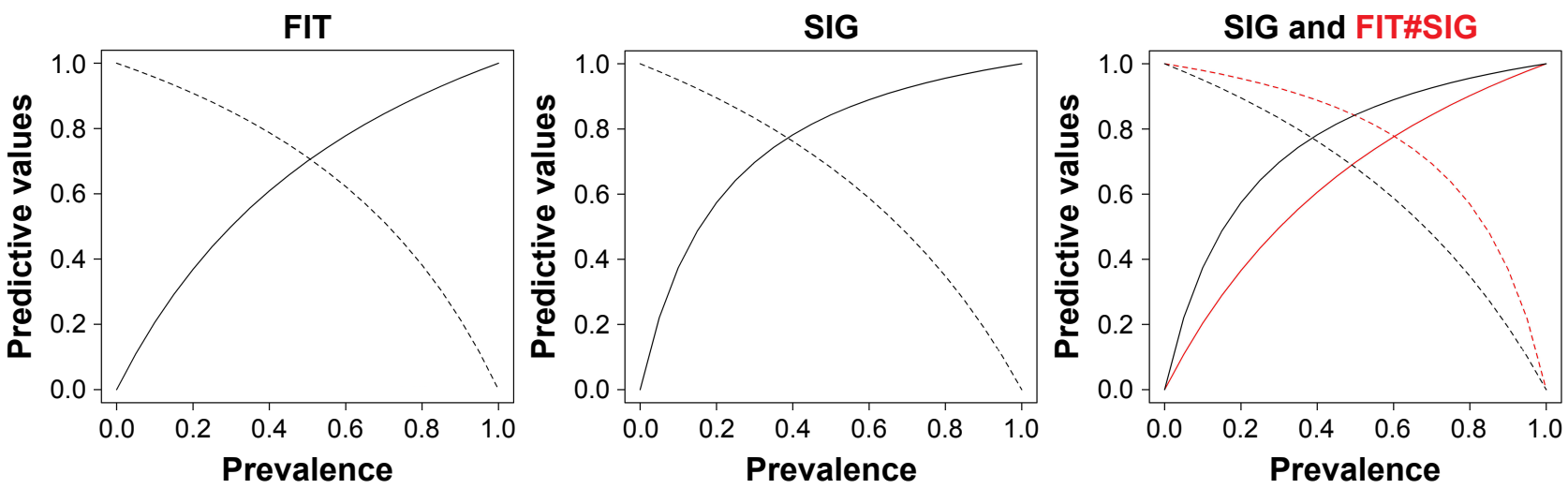

Figure 3 First stage calculated predictive values versus prevalence: FIT (left), SIG (middle), and SIG with FIT\#SIG combined test (right).

Notes: The red color text refers to the red color curves. Solid line was used for PVP and dotted line for PVN.

Abbreviations: COL, colonoscopy; FIT, fecal-immunochemical test; SIG, sigmoidoscopy; PVP, predictive value positive; PVN, predictive value negative.

probabilities in the actual population. A critical point is that, no matter how good the diagnostic tests are, prevalence and patient compliance affect the predictive value of any screening result. Therefore, the curves of predicted values versus prevalence (in a larger sense, including compliance) were drawn. Figure 3 shows predictive values versus prevalence ranging from $0 \%$ to $100 \%$ for FIT, simulated SIG following UK criteria, ${ }^{6}$ and their first-stage combination FIT\#SIG compared to SIG. As previously mentioned, the UK criteria were chosen for SIG simulation as being more conservative. The sensitivity and specificity were computed using the Equations (2') and (3'). Improved sensitivity and predictive value negative (PVN) can be observed.

Figure 4 shows predictive values versus prevalence ranging from $0 \%$ to $100 \%$ for the first-step combination FIT\#SIG, COL with sensitivity and specificity of $91.2 \%$ and $95.1 \%$, respectively, ${ }^{3}$ followed by their second-stage combination FIT\#SIG COL compared to COL. Sensitivity and specificity were computed using Equations (5') and (6'). Specificity and predictive value positive (PVP) improved, while the PVN did not fall dramatically within the first third of the prevalence range. We can see that the intersection of the two curves (ie, PVP and PVN) moved to lower prevalence values, with an improved PVP curve. For the actual values employed in this simulation, the intersection occurred at $22 \%$ prevalence with equal PVP and PVN of approximately 95\%, similar to those of COL alone at a higher prevalence level.

Table 4 presents the predictive values of the two-stage FIT\#SIG COL combination, with actual results of FIT and real SIG in arm (B) and calculated predictive values for the results with UK criteria in arm (A), as described in Figure 1.
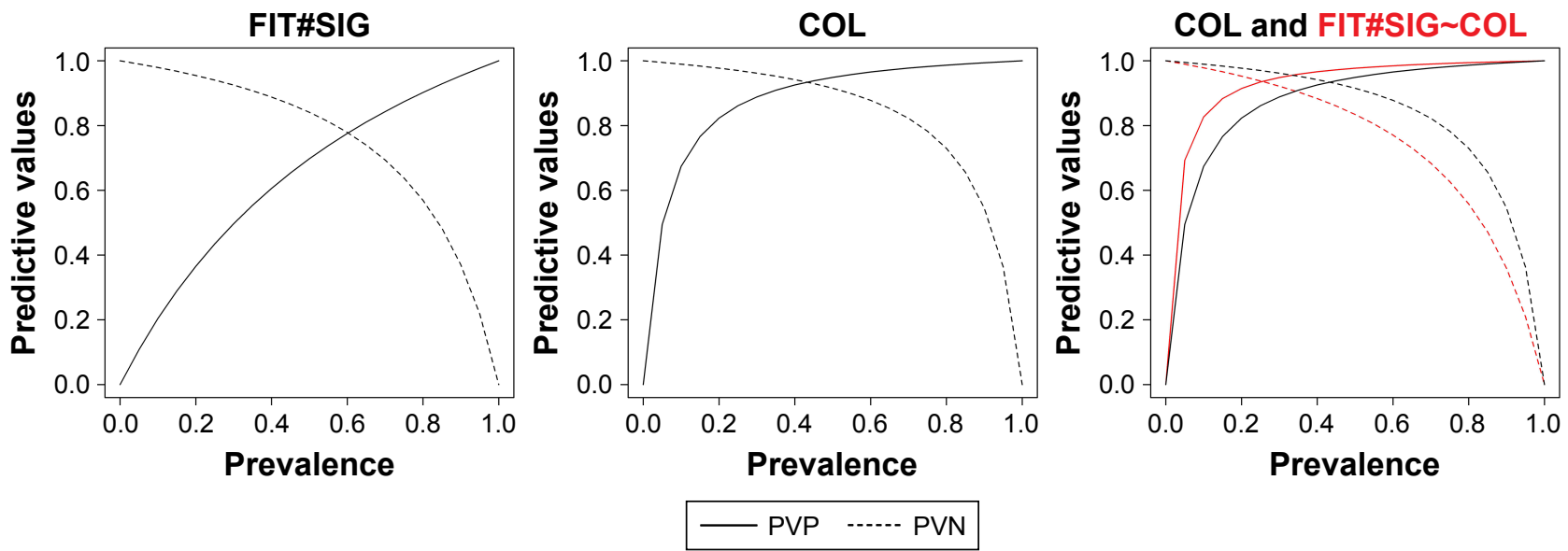

Figure 4 Second stage calculated predictive values versus prevalence: FIT\#SIG combined test (left), COL (middle), and COL with FIT\#SIG COL combined test (right). Notes: The red color text refers to the red color curves. Solid line was used for PVP and dotted line for PVN.

Abbreviations: COL, colonoscopy; FIT, fecal-immunochemical test; SIG, sigmoidoscopy; PVP, predictive value positive; PVN, predictive value negative. 
Table 4 Predictive values of FIT\#SIG COL screening strategy for colorectal neoplasia detection

\begin{tabular}{|c|c|c|c|c|}
\hline FIT\#SIG COL & PV positive ${ }^{a}(\%)$ & PV positive $(95 \% \mathrm{Cl})$ & PV negative ${ }^{a}(\%)$ & PV negative $(95 \% \mathrm{Cl})$ \\
\hline Actual - arm (B) & 36.36 & $(23.87,48.84)$ & 84.61 & $(75.24,93.98)$ \\
\hline Calculated $-\operatorname{arm}(\mathrm{A})^{\mathrm{b}}$ & 40.57 & $(34.5 I, 46.63)$ & 85.81 & $(81.5,90.12)$ \\
\hline
\end{tabular}

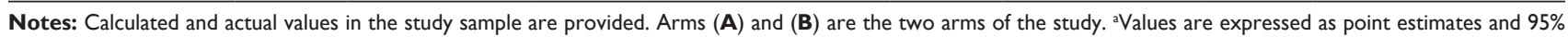

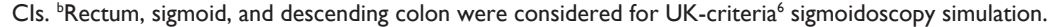

Abbreviations: COL, colonoscopy; Cl, confidence interval; FIT, fecal-immunochemical test; PV, predictive value; SIG, sigmoidoscopy.

\section{Discussion}

The results of this study demonstrate that combining flexible SIG and FIT in CRC screening would lead to better results than screening with either of them alone. Adding FIT to SIG increased sensitivity for advanced neoplasia in all the four simulating strategies evaluated. Other studies claimed similar findings, for example, using SIG in addition to FIT led to increased sensitivity for advanced proximal neoplasia by nearly $10 \%,{ }^{10}$ and adding FIT to SIG-based strategies produced a 10\%-30\% improvement in advanced right-sided neoplasia detection rate, although at the cost of a significant reduction in specificity, ${ }^{11}$ which are similar to our results.

FIT has already proved to be a cheap and harm-free procedure, which can reduce cancer mortality if used as an annual screening tool. There is conflicting data in the literature, as some authors obtained very low FIT sensitivity in detecting proximal lesions, for example, only $17 \%$ in Castro et al. ${ }^{11}$ On the other hand, all reported specificity values for FIT were reasonably acceptable, that is, a good characteristic as false positive test results lead to anxiety and unnecessary follow-up COL. It is important to note though that, in some studies, FIT proved similar sensitivity for both proximal and distal advanced neoplasia. ${ }^{12,13}$ FIT may have false negative results, but usually the missed lesions are advanced polyps, rather than cancers. Furthermore, it was observed that regular fecal-based diagnostic tests reduced the relative risk of CRC mortality by $15 \%-25 \% .{ }^{14}$ In addition, compared to $\mathrm{COL}, \mathrm{SIG}$ is a cheaper and less time-consuming procedure, it does not need sedation, and bowel preparation is simpler. However, the major disadvantage is its inability to inspect the transverse and right colon.

Combining SIG screening with regular fecal occult blood testing has been proposed as a viable option for improving the weak results of SIG in detecting proximal neoplasia. It has been shown that single SIG screening would only lead to distal CRC mortality reduction. ${ }^{15}$ In addition, when comparing the detection rates of FIT versus COL, the latter found more neoplasia and advanced adenoma, but there were no statistically significant differences between the two methods for CRC detection. ${ }^{16}$
A clear benefit of the present proposed scheme is the integration of $\mathrm{COL}$ as a second stage in a serial design. Hence, based on the good rate of PVP, when both tests from the first stage are negative, the result is negative, and further COL tests are avoided. Therefore, bearing in mind the low compliance rate for $\mathrm{COL},{ }^{17}$ the proposed design would be clearly advantageous. Moreover, the increased demand for COL units usually leads to delays in CRC diagnosis, so a high accuracy level in the detection of true negative cases in the first stage is of considerable importance. ${ }^{18}$

Test replication and standardization should improve precision; on the other hand, if the values of the diagnostic test correlated with the severity of the disease, a well-performing test for advanced illness would be less useful for identifying patients in early stages, when treatment might be most effective. ${ }^{19}$ A surveying methodology for the systematic investigation of gastrointestinal disorders has also been proposed. ${ }^{20}$

Taking all this context into account, the proposed pilot scheme is also advantageous for multiple testing, since it helps in the making of unequivocal diagnosis by altering the posterior probabilities in a predictable fashion, that is, parallel testing results with decreased false negative rate, while serial testing leads to decreased false positive rate. Moreover, there is less unnecessary anticipated discomfort, lowering the delays and increasing compliance. In addition, as the information is clearer, available, and explicable to all the stakeholders, it is easier to assess and revise assumptions when deciding on each practice approach. Moreover, patients' compliance increases when a concrete investigation scheme is provided.

A limitation of this study is that all the estimates, except for COL sensitivity and specificity, were not independent, but derived from the study population data and were dependent on the existing knowledge and technology. Therefore, more research should be conducted to get independent estimates of the prior probabilities.

Our study was also limited to a CRC screening single round. In addition, there was a rather small number of patients with a high mean age (63.65 years) and female predominance 
(57.1\%). Nevertheless, this might not be a hindrance, as it is known that the prevalence of proximal colonic neoplasia increases with age and is higher in females than in males. ${ }^{21}$ Also, patients were recruited in a Gastroenterology outpatient clinic, all of them being symptomatic (ie, not a general screening population), although none with cancer referral symptoms (eg, unexplained rectal bleeding, iron deficiency anemia, or changes in their bowel habit). FIT was performed in clinical conditions, therefore good performance results were obtained, which might not be entirely reproducible in less controlled conditions. Employing the UK flexible SIG criteria $^{9}$ in simulation was aimed at compensating for this potential difficulty.

Although the compliance rate was rather poor (ie, only 108 out of the 206 FIT-positives agreed to undergo a subsequent $\mathrm{COL}$ ), the data were similar to those reported elsewhere, ${ }^{17-19}$ and justified the search for a friendlier and less inconvenient approach toward the patient. Combined with lower costs, this could achieve better screening compliance. ${ }^{17}$

Another aspect to consider is that different strategies to identify COL referrals have been used in order to limit and better target its employment as an investigation procedure. When choosing a SIG strategy, one must bear in mind the need to balance between detecting many lesions and increasing the COL burden. The values obtained in our study for simulated SIG sensitivity and specificity were similar with those obtained in other studies. While the criteria proposed in the UK Flexible Sigmoidoscopy trial seemed to be the most appropriate in terms of saving resources, the USPLCO trial was the only one to demonstrate a statistically significant reduction in colon cancer incidence. ${ }^{17,22}$ The mean values to increase the prior probability in the screening population include explicit referral criteria to be followed by the primary care physicians, risk-scoring based on symptoms and medical best practice recommendations. ${ }^{18,23}$

With the proposed screening scheme, at a prevalence of $\sim 3 \%-4 \%$, the percent of negative subjects who would avoid the unnecessary invasive procedure of COL is between $95 \%$ and $99 \%$, so better screening compliance should be expected.

\section{Conclusion}

Flexible SIG and FIT can play an important role in CRC screening, since they are less challenging in terms of the demands placed on resources on the one hand, and less invasive on the other, and so are more affordable when applied on a large scale. In this context, test combination significantly improves neoplasia detection, increasing sensitivity and specificity, as well as predictive values in usual screening scenarios. Moreover, on the patient side, having a clear scheme for the screening stages and actual predictive values, there is a greater likelihood of both informed consent and compliance with the physician's recommendations.

\section{Acknowledgment}

The authors gratefully acknowledge the contribution of the professionals from the County Hospital of Arad for their valuable contribution to this study.

\section{Author contributions}

Conceived and designed the study: DI, MF, DL, and EM. Conducted the study, performed the medical investigations, and collected the data: DI, AB, and BPM. Analyzed and interpreted data: DI, MF, DL, and EM. Wrote the paper: DI, MF, DL, and EM. All authors contributed toward data analysis, drafting and revising the paper and agree to be accountable for all aspects of the work. All authors reviewed and approved the final manuscript.

\section{Disclosure}

The authors report no conflicts of interest in this work.

\section{References}

1. Ferlay J, Shin H, Bray F, Forman D, Mathers C, Parkin D. Estimates of worldwide burden of cancer in 2008. Int J Cancer. 2010;127(12): 2893-2917.

2. Lansdorp-Vogelaar I, Knudsen AB, Brenner H. Cost-effectiveness of colorectal cancer screening. Epidemiol Rev. 2011;33:88-100.

3. Zauber AG, Lansdorp-Vogelaar I, Knudsen AB, Wilschut J, van Ballegooijen M, Kuntz KM. Evaluating test strategies for colorectal cancer screening: a decision analysis for the U.S. preventive services task force. Ann Intern Med. 2008;149(9):659-669.

4. Pignone M, Saha S, Hoerger T, Mandelblatt J. Cost-effectiveness analyses of colorectal cancer screening: A systematic review for the U.S. preventive services task force. Ann Intern Med. 2002;137(2):96-104.

5. Schoen RE, Pinsky PF, Weissfeld JL, et al. Colorectal-cancer incidence and mortality with screening flexible sigmoidoscopy. $N$ Engl J Med. 2012;366(25):2345-2357.

6. Atkin WS, Edwards R, Kralj-Hans I, et al; UK Flexible Sigmoidoscopy Trial Investigators. Once-only flexible sigmoidoscopy screening in prevention of colorectal cancer: a multicentre randomised controlled trial. Lancet. 2010;375(9726):1624-1633.

7. Segnan N, Armaroli P, Bonelli L, et al; for SCORE Working Group: Once-only sigmoidoscopy in colorectal cancer screening: follow-up findings of the italian randomized controlled trial - SCORE. J Natl Cancer Inst. 2011;103(17):1310-1322.

8. Bretthauer M, Gondal G, Larsen I, et al. Design, organization and management of a controlled population screening study for detection of colorectal neoplasia: attendance rates in the NORCCAP study (norwegian colorectal cancer prevention). Scand J Gastroenterol. 2002; 37(5):568-573.

9. Ait Ouakrim D, Pizot C, Boniol M, et al. Trends in colorectal cancer mortality in Europe: retrospective analysis of the WHO mortality database. BMJ. 2015;351:h4970. 
10. Kato J, Morikawa T, Kuriyama M, et al. Combination of sigmoidoscopy and a fecal immunochemical test to detect proximal colon neoplasia. Clin Gastroenterol Hepatol. 2009;7(12):1341-1346.

11. Castro I, Estevez P, Cubiella J, et al. Diagnostic performance of fecal immunochemical test and sigmoidoscopy for advanced right-sided colorectal neoplasms. Dig Dis Sci. 2015;60(5):1424-1432.

12. de Wijkerslooth T, Stoop E, Bossuyt P, et al. Immunochemical fecal occult blood testing is equally sensitive for proximal and distal advanced neoplasia. Am J Gastroenterol. 2012;107(10):1570-1578.

13. Cubiella J, Salve M, Diaz-Ondina M, et al. Diagnostic accuracy of the faecal immunochemical test for colorectal cancer in symptomatic patients: comparison with nice and sign referral criteria. Colorectal Dis. 2014;16(8):273-282.

14. Hewitson P, Glasziou P, Irwig L, Towler B, Watson E. Screening for colorectal cancer using the faecal occult blood test, hemoccult. Cochrane Database Syst Rev. 2011;(1):CD001216.

15. Brenner H, Stock C, Hoffmeister M. Effect of screening sigmoidoscopy and screening colonoscopy on colorectal cancer incidence and mortality: systematic review and meta-analysis of randomised controlled trials and observational studies. BMJ. 2014;348:g2467.

16. Salas D, Vanaclocha M, Ibanez J, et al. Participation and detection rates by age and sex for colonoscopy versus fecal immuno-chemical testing in colorectal cancer screening. Cancer Causes Control. 2014;25(8): 985-997.
17. Schoen RE, Machicado JD. Detection of advanced neoplasia with FIT versus flexible sigmoidoscopy versus colonoscopy: more is more. Dig Dis Sci. 2015;60(5):1123-1125.

18. Vega P, Valentin F, Cubiella J. Colorectal cancer diagnosis: Pitfalls and opportunities. World J Gastrointest Oncol. 2015;7(12):422-433.

19. Bujanda L, Sarasqueta C, Castells A, et al; EUSCOLON Study Investigators. Colorectal cancer in a second round after a negative faecal immunochemical test. Eur J Gastroenterol Hepatol. 2015;27(7):813-818.

20. Yan X, Wang R, Zhao Y, et al. Systematic investigation of gastrointestinal diseases in China (SILC): validation of survey methodology. BMC Gastroenterol. 2009;9:86.

21. Nawa T, Kato J, Kawamoto H, et al. Differences between right-and left-sided colon cancer in patient characteristics, cancer morphology and histology. J Gastroenterol Hepatol. 2008;23(3):418-423.

22. Castells A, Bessa X, Quintero E, et al. Risk of advanced proximal neoplasms according to distal colorectal findings: comparison of sigmoidoscopy-based strategies. J Natl Cancer Inst. 2013;105(12): 878-886.

23. Sive Droste J, Macken E, Peeters M. Screening for colorectal cancer: when, how, and by whom? Curr Colorectal Cancer Rep. 2013;9(1): $17-23$.
OncoTargets and Therapy

\section{Publish your work in this journal}

OncoTargets and Therapy is an international, peer-reviewed, open access journal focusing on the pathological basis of all cancers, potential targets for therapy and treatment protocols employed to improve the management of cancer patients. The journal also focuses on the impact of management programs and new therapeutic agents and protocols on

\section{Dovepress}

patient perspectives such as quality of life, adherence and satisfaction. The manuscript management system is completely online and includes a very quick and fair peer-review system, which is all easy to use. Visit http://www.dovepress.com/testimonials.php to read real quotes from published authors. 